\title{
A Study of the Wave Transformation Passing over an Artificial Reef Using SPH Method*
}

\author{
Tamon SUWA**, Tomokazu NAKAGAWA*** and Keisuke MURAKAMI ${ }^{\dagger}$ \\ ** FUJITSU Limited \\ 4-1-1, Kamikodanaka, Nakahara-ku, Kawasaki, Kanagawa, Japan \\ E-mail: suwa.tamon@jp.fujitsu.com \\ *** Kobe Steel Ltd.
}

1-5-5, Takatsukadai, Nishi-ku, Kobe, Hyogo, Japan

University of Miyazaki

1-1, Gakuen Kibanadai-nishi, Miyazaki-shi, Miyazaki, Japan

\begin{abstract}
We present some improvement methods for Smoothed Particle Hydrodynamics (SPH) method to obtain accurate solutions for surface wave propagation problems. To verify the accuracy of this method, a water tank test of wave propagation on an artificial reef is performed. As a result, simulated wave height histories agreed well with measured ones even in the case of wave breaking situations in which deformation of wave takes place strongly.
\end{abstract}

Key words : Computational Method, Numerical Simulation, Free Surface Flow, Fluid Dynamics, Marine Structures

\section{Introduction}

Smoothed Particle Hydrodynamics (SPH) method is a particle method for solving partial differential equations ${ }^{(1)}$. While it has been used highly successfully in astrophysical applications, in recent years it has been developed as a method for solving incompressible flows.

There is a problem that the wave height attenuates when the propagation of the surface wave is simulated for a long duration time by using the SPH method ${ }^{(2)}$. To solve this problem, we have made several improvements to "conventional" SPH method. Definitions of the conventional method will be described in $\$ 2$. For the verification of the improved method, we conducted the surface wave propagation test using water tank with simple slope. As a result, measured wave heights agreed well with the simulated values ${ }^{(6),(7)}$.

The objective of this study is to confirm the reliability of the surface wave propagation analysis in the situation where complicated sea bed topography exists. For this purpose, we treat wave transformation passing over an artificial reef. We compare numerical computation results and hydraulics model tests containing an artificial reef, and validate reproducibility of complicated surface wave propagation phenomena simulated by the SPH method.

An artificial reef is one of the submerged shore protection facilities, which is used on eroded beaches and protect shore by dissipation of wave. Complicated wave phenomena are observed near the reef, such as, wave breaking at front of the reef, and wave reformation at back of the reef, which are hardly to be reproduced by numerical simulations.

In this paper, a numerical method is described in $\S 2$. Also, in $\S 3$, we show the result of our simulation of water tank with an artificial reef, and compare the results and experimental data. 


\section{Numerical Procedures}

\subsection{Discretization method of the SPH scheme}

The interpolated value of a function $A$ of a particle $a$ using the SPH methodology is:

$$
A_{a}=\sum_{b} \frac{m_{b}}{\rho_{b}} A_{b} W_{a b}
$$

where $a, b$ are indexes of the particles, and $m$ and $\rho$ are mass and density of the particles, respectively. $W_{a b}=W\left(\vec{r}_{b}-\vec{r}_{a}, h\right)$ is an interpolation kernel function of radius $2 h$. The sum is over all particles $b$ within a radius $2 h$ from the particle $a$.

The gradient of the function $A$ is given by

$$
\nabla A_{a}=\sum_{b} \frac{m_{b}}{\rho_{b}} A_{b} \nabla W_{a b}
$$

As a kernel function we employ the quintic spline ${ }^{(3)}$.

$$
W(r, h)=\left\{\begin{array}{ll}
\alpha\left(1-\frac{q}{2}\right)^{4}(2 q+1) & (0 \leq q \leq 2) \\
0 & \text { (otherwise) }
\end{array},\right.
$$

where $q=r / h$, and $\alpha$ is a normalization constant.

In the conventional method, the cubic spline is used, instead of quintic.

$$
W(r, h)=\alpha \begin{cases}1-\frac{3}{2} q^{2}+\frac{3}{4} q^{3} & (0 \leq q \leq 1) \\ \frac{1}{4}(2-q)^{3} & (1 \leq q \leq 2), \\ 0 & \text { (otherwise) }\end{cases}
$$

We use a form of the SPH momentum equation as follow ${ }^{(4)}$.

$$
\begin{aligned}
\frac{d \vec{v}_{a}}{d t}= & -\sum_{b} m_{b}\left(\frac{p_{b}+p_{a}}{\rho_{b} \rho_{a}}\right) \nabla_{a} W_{a b} \\
& +\sum_{b} m_{b}\left(\frac{\beta_{\mathrm{av}} \phi^{2}}{\bar{\rho}_{a b}}\right) \nabla_{a} W_{a b} \\
& +\vec{g}
\end{aligned}
$$

Here, $\vec{v}, p$, and $\vec{g}$ are the velocity, the pressure, and the gravitational acceleration, respectively. $\bar{\rho}_{a b}$ is the average of the densities of particles $a$ and $b$. Second term of right hand side of the equation (5) represents artificial viscosity, where $\phi$ is defined as follows.

$$
\phi=\left\{\begin{array}{ll}
\frac{h \vec{r}_{b a} \cdot \vec{v}_{b a}}{r_{b a}+\eta^{2}} & \left(\vec{r}_{b a} \cdot \vec{v}_{b a}<0\right) \\
0 & \left(\vec{r}_{b a} \cdot \vec{v}_{b a} \geq 0\right)
\end{array},\right.
$$

where $\vec{r}_{b a}=\left(\vec{r}_{b}-\vec{r}_{a}\right)$, and $\vec{v}_{b a}=\left(\vec{v}_{b}-\vec{v}_{a}\right) . \beta_{\mathrm{av}}$ and $\eta$ are tuning parameters, and we set the values as $\beta_{\text {av }}=0.1$ and $\eta=0.01$.

In the conventional method, The momentum equation is as follows.

$$
\frac{d \vec{v}_{a}}{d t}=-\sum_{b} m_{b}\left(\frac{p_{b}}{\rho_{b}^{2}}+\frac{p_{a}}{\rho_{a}^{2}}+\Pi_{a b}+\frac{\beta_{\mathrm{av}} \phi^{2}}{\bar{\rho}_{a b}}\right) \vec{\nabla}_{a} W_{a b}+\vec{g}
$$

Laminar viscosity used in Ref (5) is introduced.

$$
\Pi_{a b}=-\frac{\xi}{\rho_{a} \rho_{b}} \frac{4 \mu_{a} \mu_{b}}{\mu_{a}+\mu_{b}} \frac{\vec{v}_{a b} \cdot \vec{r}_{a b}}{r_{a b}^{2}+\eta^{2}}
$$

$\mu$ is viscosity, and $\xi$ is a tuning parameter. We use a value of $\xi=4.96333$ suggested by (5).

The equation of continuity is as follows:

$$
\frac{d \rho_{a}}{d t}=\sum_{b} m_{b}\left(\vec{v}_{a}-\vec{v}_{b}\right) \vec{\nabla}_{a} W_{a b}
$$

The pressure is given by the following equation of state.

$$
p=c_{0}^{2}\left(\rho-\rho_{0}\right),
$$

where $\rho_{0}$ and $c_{0}$ are initial density and sound speed, respectively. In this study, $\rho_{0}$ and $c_{0}$ are set to $1 \mathrm{~g} / \mathrm{cm}^{3}$ and $50 \mathrm{~m} / \mathrm{sec}$, respectively. 


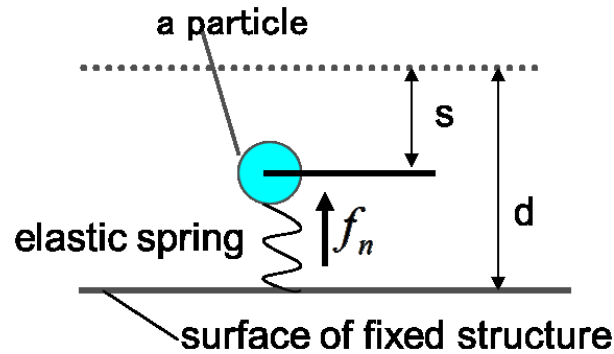

Fig. 1 Conceptual diagram of boundary model at surface of fixed structure.

\subsection{Time integration scheme}

Following symplectic Euler algorithm is used as a time stepping scheme, since it is comparatively superior in the energy preservation.

( 1 ) The acceleration at the step $n, d \vec{v}_{a} / d t=\vec{F}_{a}^{n}$ is calculated using equation (5).

( 2 ) Update the velocity: $\vec{v}_{a}^{n+1}=\vec{v}_{a}^{n}+\Delta t \vec{F}_{a}^{n}$

( 3 ) The time derivative of the density at the step $n+1, d \rho_{a} / d t=D_{a}^{n+1}$ is calculated using equation (9)

( 4 ) Update the density: $\rho_{a}^{n+1}=\rho_{a}^{n}+\Delta t D_{a}^{n+1}$

( 5 ) Update the position: $\vec{r}_{a}^{n+1}=\vec{r}_{a}^{n}+\Delta t \vec{v}_{a}^{n+1}$

Time step size $\Delta t$ is calculated using;

$$
\Delta t=0.1 \times \min \left(\frac{h}{c_{0}}, \sqrt{\frac{h}{g}}\right) .
$$

Leap-frog method is used as a time stepping scheme in the conventional method.

\subsection{Smoothing of the density and the pressure}

In order to avoid unrealistic oscillation of pressure, the density is smoothed according to the following equation

$$
\rho_{a}^{\text {new }}=\frac{\sum_{b} m_{b} W_{a b}}{\sum_{b}\left(m_{b} / \rho_{b}\right) W_{a b}} .
$$

If a particle has sufficient neighboring particles, the denominator should be unity. On the other hand, at the location where particles have insufficient neighbors (such as surface regions), this correction compensate deficiency of neighboring particles. Pressure is also updated according to the equation of state (10). The smoothing was performed once a 20 steps in the present study.

\subsection{Boundary condition at fixed structure}

In our simulation, we consider a layer of width $d$ on surface of fixed structure such as slope and artificial reef. When a particle moves to inside of the layer, the particle is exerted repulsive force. The repulsive force $f_{n}$ is defined as a similar form of elastic spring $f_{n}=k s$, where $k$ is a spring constant, $s$ is distance from surface of the layer. The spring constant $k$ is defined such that the kinetic energy of a particle with velocity $c_{0}$ is equal to the elastic strain energy when $s=d$, i.e. $m c_{0}^{2} / 2=k d^{2} / 2$. The acceleration of the particle is then expressed as

$$
\dot{v}_{n}=\frac{\left(\beta c_{0}\right)^{2}}{d^{2}} s
$$

where $\beta$ is a tuning parameter of order of unity.

\subsection{Parallel computation}

The simulation program is performed with distributed memory type parallelization by Message Passing Interface (MPI). The domain decomposition technique is used, in which 


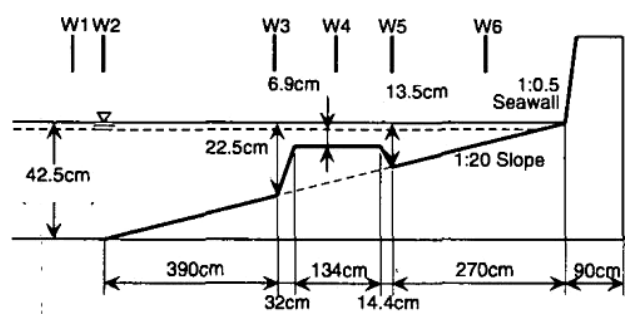

Fig. 2 Water tank used by the hydraulics model test.

Table 1 The hydraulic model test conditions

\begin{tabular}{|l|c|c|}
\hline & wave height $(\mathrm{m})$ & period $(\mathrm{sec})$ \\
\hline caseA1 & 0.02 & 1.5 \\
\hline caseA2 & 0.03 & 2.0 \\
\hline caseA3 & 0.04 & 1.5 \\
\hline caseA4 & 0.06 & 2.0 \\
\hline
\end{tabular}

each node takes charge of the divided spatial domain and performs only calculation of the particles contained in the domain. Since particle interactions are limited inside the region with the smoothing length of $2 h$, most calculations are independently performed within a node. However, since the information on the particles contained in an adjacent node is required for the particles which locate in a domain boundary, it is needed to communicate mutually the information on the particles at the node boundary.

\subsection{Setting of the numerical simulation}

In order to validate reproducibility of complicated surface wave propagation phenomena simulated by the SPH method, we compare numerical computation results with hydraulics model tests by Oki, Murakami, and Mase ${ }^{(8)}$. The water tank used by the experiment is shown in Fig. 2. In the experiment, wave gauges are set up at the positions indicated by W1-W6.

Four kinds of sinusoidal wave described in Table 1 are set up as input. In the simulation, virtual wave maker is set at $\mathrm{W} 1$ to generate sinusoidal waves. Motion of the wave maker is set by the following equation;

$$
\dot{x}=\frac{\omega}{\bar{A}}\left(2 \eta_{D}-\eta_{0}\right),
$$

where $\dot{x}$ is velocity of the wave maker, $\eta_{D}$ is target wave height, $\eta_{0}$ is simulated wave height at the front of the wave maker, and $\omega$ is angular frequency. Also,

$$
\bar{A}=\frac{2 \sinh ^{2} K H}{K H+\sinh K H \cosh K H},
$$

where $H$ is still water height, $K$ is wave number. The wave number $K$ can be obtained from the following equation

$$
\frac{\omega^{2}}{g}=K \tanh K H \text {. }
$$

Number of particles employed in the simulation is about 280,000. Initial particle distance is $2.5 \times 10^{-3} \mathrm{~m}$ and the radius of the kernel function $(2 h)$ is $6.25 \times 10^{-3} \mathrm{~m}$.

\section{Results}

\subsection{Wave breaking and reformation on the edge of the reef}

Fig. 3 and 4 show distribution of fluid particles near the front of the artificial reef and the back of the reef, respectively. The wave condition is case A4. Small arrows correspond to the velocity vector of the particles and the color indicates the magnitude of the velocity. It is seen that the wave breaking occurs at the front of the reef in Fig. 3. Also, in Fig. 4, broken wave reforms at the back of the reef. These results well correspond with the hydraulic model test. 


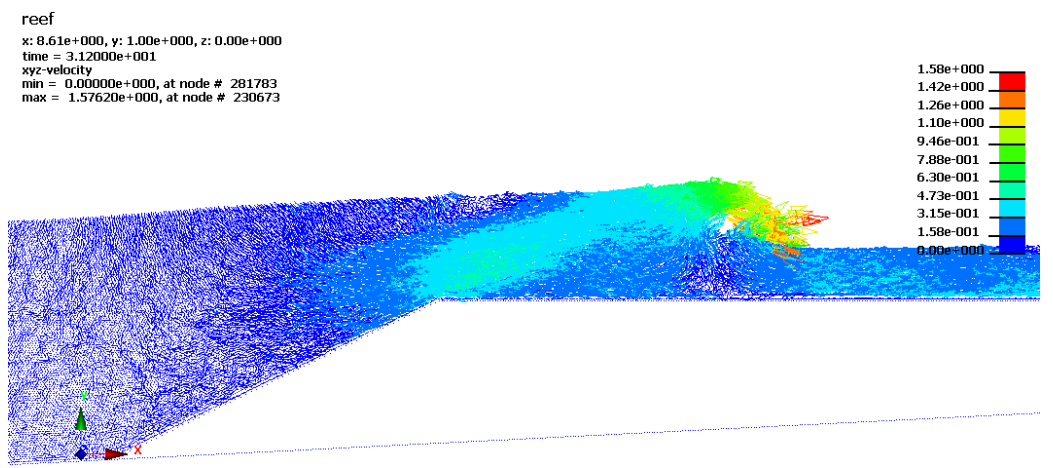

Fig. 3 Front of the reef

reef

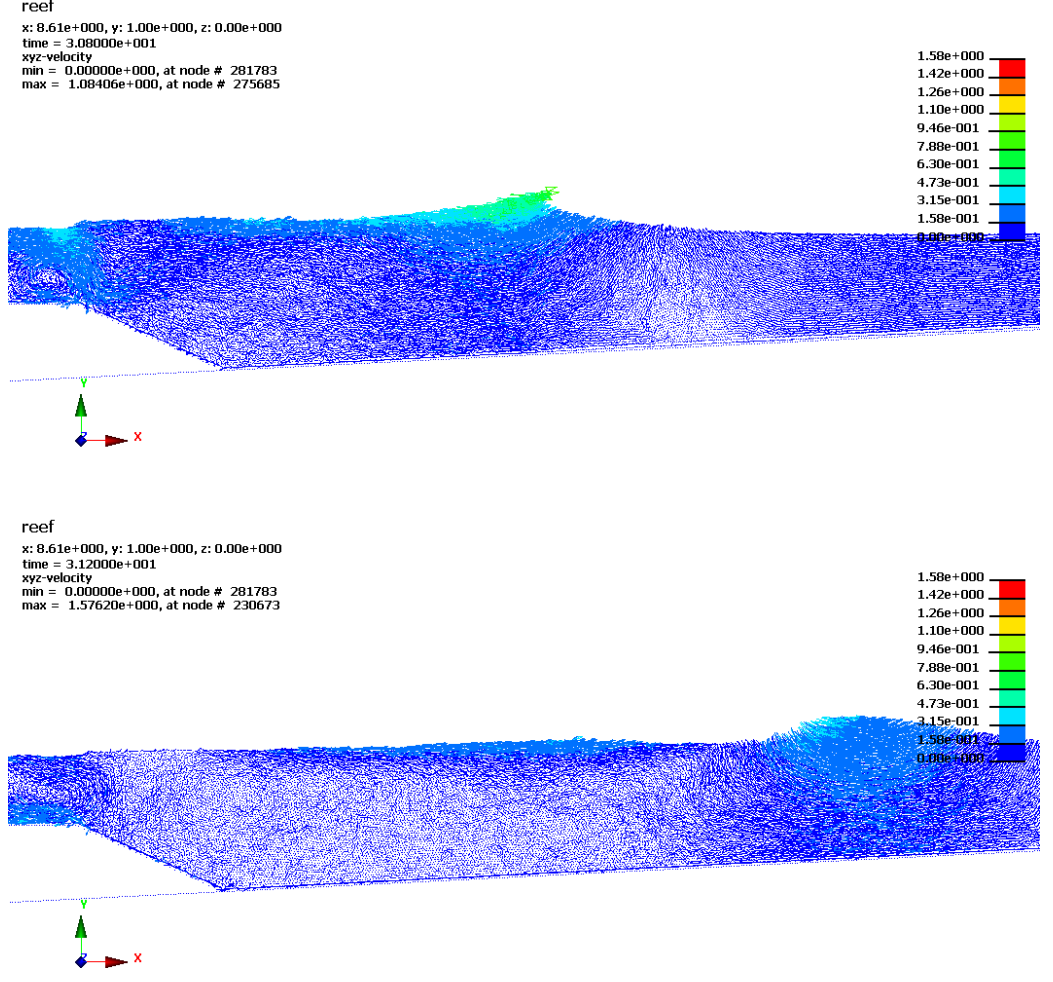

Fig. 4 Back of the reef 


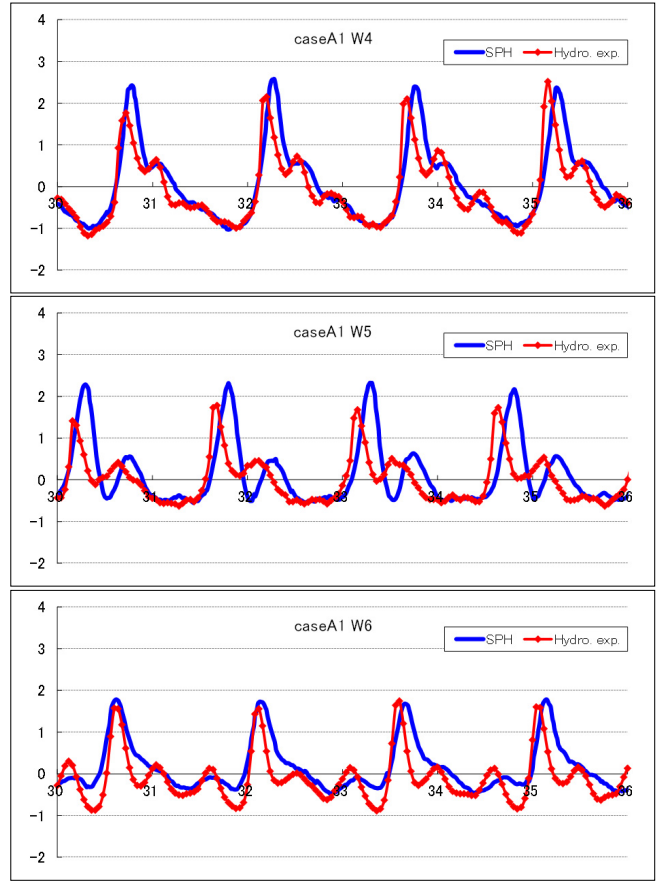

Fig. 5 The wave height at the point 4,5 , and 6 normalized by the value at W2 as a function of the time (caseA1)

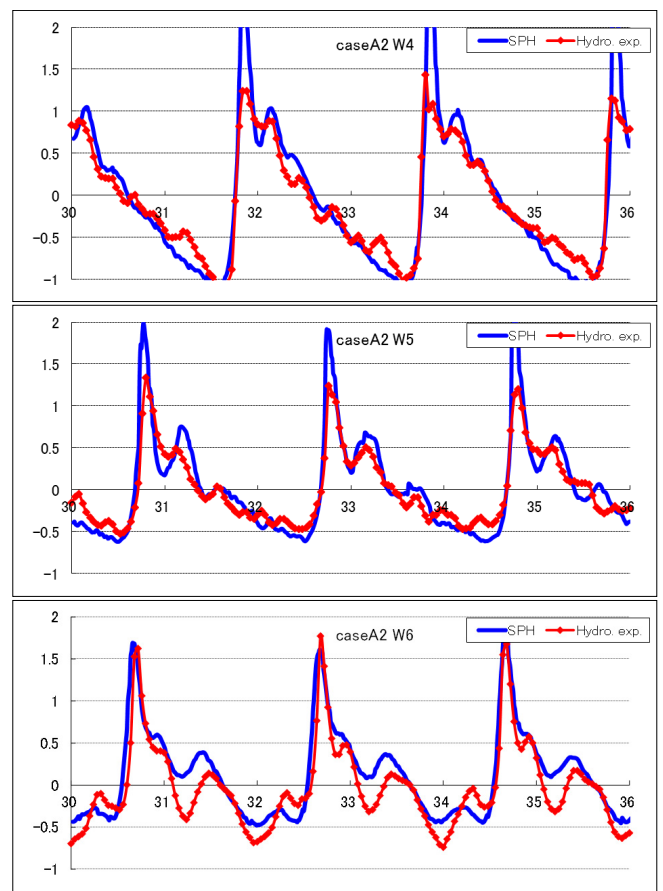

Fig. 6 The wave height at the point 4,5, and 6 normalized by the value at W2 as a function of the time (caseA2) 

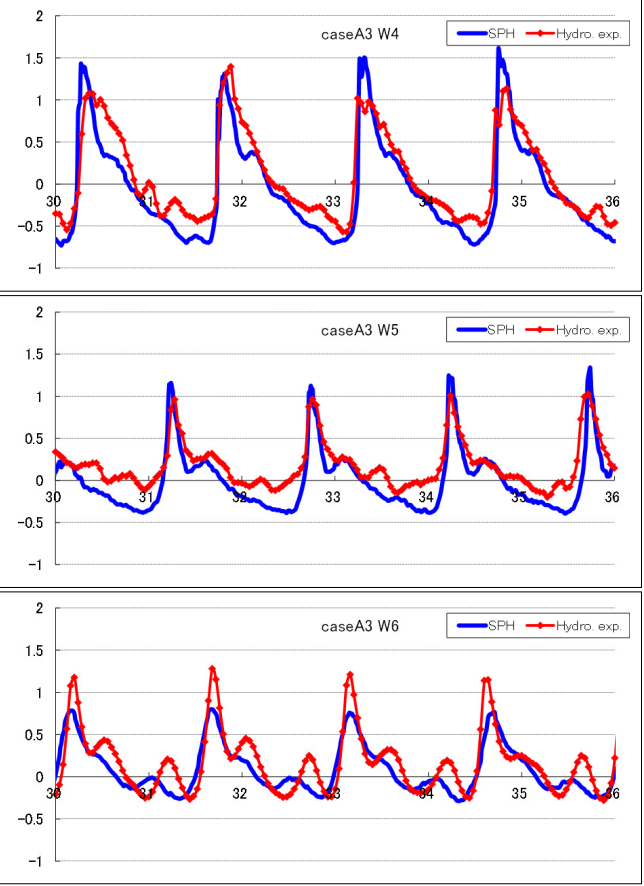

Fig. 7 The wave height at the point 4,5 , and 6 normalized by the value at W2 as a function of the time (caseA3)

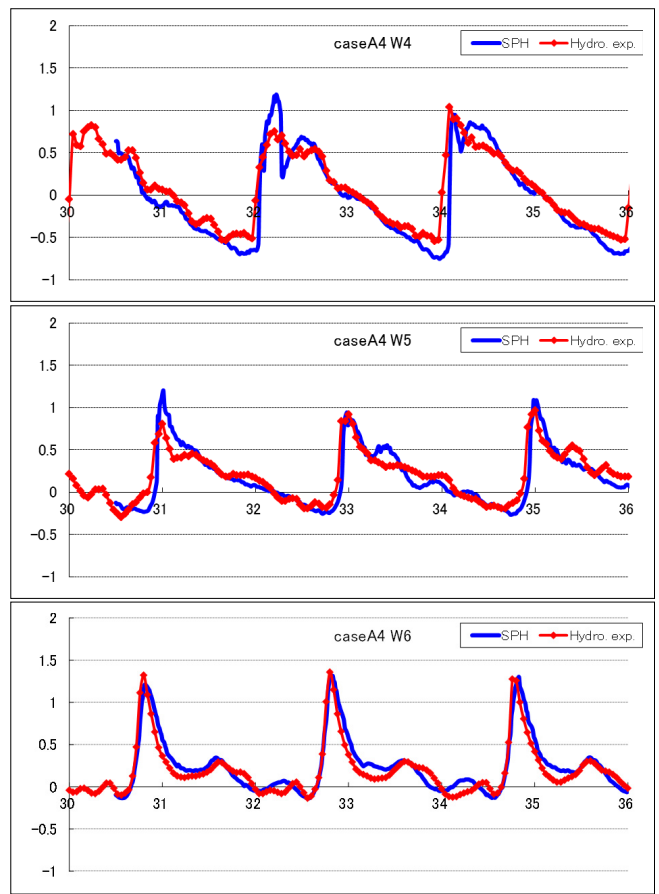

Fig. 8 The wave height at the point 4,5 , and 6 normalized by the value at $\mathrm{W} 2$ as a function of the time (caseA4) 


\subsection{Comparison of the wave height history}

In Fig. 5-8, we show wave height histories of the case A1-A4 at the points W4, W5, and W6, respectively. The wave height is normalized by the mean wave height at the point W2. The red curves indicate the experimental data, and the blue ones are the results of numerical simulation.

In case A1 and A2, while the simulated wave height at W5 are larger than those of the hydraulics model test, the wave height at W6 of the experiment and the simulation are in good agreement. On the other hand, in case A3, the simulated wave height is well in agreement with the experiment at W5, and it is smaller than the experiment at W6. It can be seen that the energy dissipation (it can be thought as the effect of artificial viscosity) of a wave has occurred from W5 toward W6 in these cases. In case A4, the agreements are very well at all of W4-W6. This suggest that it is related to the size of wave height, and the reproducibility of a wave height history. It may be required for the reproducibility of a wave height history that wave height is large to some extent, which might be related to the particle resolution.

\section{Conclusions}

(1) We presented an improved SPH method using the symplectic time integration scheme and the equation of motion derived from the law of the energy conservation.

(2) Wave heights simulated by this method agreed well with measured ones obtained by the water tank experiments with the artificial reef. Especially, highly nonlinear phenomenon, i.e. wave breaking and recovering on the reef can be predicted precisely.

\section{Acknowledgments}

The authors are grateful to Prof. Mase for the water tank experimental data.

\section{References}

( 1 ) Monaghan, J.J. :Smoothed Particle Hydrodynamics, Annu. Rev. Astron. Astrophys., Vol.30, pp.543-574, 1992

( 2 ) Guilcher, P.-M., Ducrozet, G., Alessandrini, B., and Ferrant, P. :Water wave propagation using SPH method, Proc. of 2nd Int. SPHERIC Workshop, Spain, pp.119-124, 2007

( 3 ) Gesteria, M.G., Rogers, B.D., Dalrymple, R.A., Crespo, A.J.C., Narayanaswamy, M. :User Guide for the SPHysics code, September, 2010, url: http://wiki.manchester.ac.uk/sphysics

( 4 ) Fang, J., Parriaux, A., Rentschler, M., and Ancey, C. :Improved SPH methods for simulating free surface flows of viscous fluids, Applied Numerical Mathematics, Vol.59, pp.251-271, 2009

( 5 ) Cleary, P.W. :Modelling confined multi-material heat and mass flows using SPH, Appl. Math. Modelling, vol.22, pp.981-993, 1998

( 6 ) Suwa, T., Kazama, M., Nakagawa, T., and Kataoka, Y. : Surface Wave Propagation Analysis by SPH Method (in Japanese), Proceedings of the Conference on Computational Engineering and Science, Vol.16, D-1-3, 2011

( 7 ) Suwa, T., Nakagawa, T., and Murakami, K. : A Study of the Water Wave Propagation over an Artificial Reef using SPH Method (in Japanese), Proceedings of the Conference on Computational Engineering and Science, Vol.17, C-2-6, 2012

( 8 ) Oki, K., Murakami, K., and Mase, H.: Wave Transformation Passing Over an Artificial Reef with Very Shallow Submerged Depth of Crown, Proceedings of Coastal Engineering, JSCE, Vol.49, pp.41-45. 2002 (in Japanese) 